\title{
Índice de percepción local de mejora del combate al rezago social: análisis de las centrales eólicas en el Istmo de Tehuantepec, Oaxaca
}

\author{
Local perception index of improvement in the struggle against social \\ backwardness: analysis of wind farms in the Isthmus of Tehuantepec, Oaxaca
}

\author{
José Domingo Rafael Castañeda Olvera* \\ Tamar Zehla JimÉnez VelázQuez ** \\ Raúl Ramírez Ramírez *** \\ doi.org/10.29043/liminar.v20i1.891
}

Resumen: El objetivo de este artículo es proponer un nuevo índice local de percepción de mejora del combate al rezago social en la región del Istmo de Tehuantepec, Oaxaca, como resultado de la puesta en marcha de centrales eólicas. Nos apoyamos en las dimensiones analíticas holísticas propuestas por el Metabolismo Social para cuantificar las interacciones biofísicas entre sociedad y medio ambiente, en el uso de Sistemas de Información Geoespacial, así como en datos arrojados por el Coneval e INEGI respecto al rezago social. Concluimos que la percepción de mejora sobre este índice en la región no ha incrementado con la presencia de estos megaproyectos ya que estos no mitigan los impactos ecosistémicos ni coadyuvan al combate de tal fenómeno.

Palabras clave: rezago social, metabolismo social, centrales eólicas, pueblos originarios, sistemas de información geoespacial.

Abstract: The aim of this study is to analyze how people struggle against social backwardness in the Isthmus of Tehuantepec, Oaxaca, in response to the installation of wind farms in the region. We rely on the holistic analytical dimensions proposed by Social Metabolism to quantify biophysical interactions between society and environmental, while making use of Geospatial Information Systems and data produced by Coneval and INEGI regarding social backwardness. We conclude that, in the region, the perception of improvement in the social backwardness index has not increased with the presence of these megaprojects, given that they do not mitigate ecosystem impacts or social backwardness.

Key words: socio-backwardness, social metabolism, wind farms, indigenous people, geospatial information systems.

Recibido: 2 de junio de 2020

Aceptación: 14 de junio de 2021

Publicación: 21 de octubre de 2021
* Universidad Tecnológica Fidel Velázquez, México rafaelcastaneda7@gmail.com (iD 0000-0002-3930-1674

** Investigadora independiente, México sociologa.ambiental@gmail.com (iD) 0000-0003-2937-9985

*** Investigador independiente, México raul_ramirez.r@hotmail.com (iD) $0000-0002-7816-6284$ 


\section{Introducción}

$\mathrm{E}$ 1 combate al rezago social ha sido profundamente reflexionado por investigadores y organismos internacionales (House of Commons, 2010; Kozulj, 2009); esto se ha visto reflejado en el esfuerzo de gobernanza global plasmado en los Objetivos de Desarrollo Sostenible (ODS) de Naciones Unidas, iniciativa que resume varios de los aspectos que trataremos en esta investigación como los siguientes: (1) la mayoría de las personas que viven por debajo del umbral de la pobreza lo hacen en tres regiones: África Subsahariana, Asia meridional y América Latina; en ellas, (2) los índices de rezago social más altos se localizan en áreas rurales, donde hay una alta dependencia humana de los recursos de la naturaleza para sobrevivir, pero también donde (3) se concentran los niveles más altos de extracción industrial de recursos naturales debido a la gran dependencia energética de los recursos fósiles que caracteriza el estilo de vida moderno; por ello, una de la metas de los ODS es (4) lograr una reconversión acelerada de la matriz energética tendiente hacia las energías renovables y energías limpias, para con ello (5) atacar de manera frontal el rezago social en las regiones con poblaciones más vulnerables a nivel global. ${ }^{1}$

Datos de Naciones Unidas aseguran que el 60 \% de la emisión de Gases de Efecto Invernadero (GEI) global provienen de la generación de energía debido a que solo el $17 \%$ de esta es producida a través de fuentes renovables; además, en el mundo el $15 \%$ de la población no tiene acceso a energía segura, y la cifra se eleva hasta un $35 \%$ si se incluye a las poblaciones más vulnerables y con mayor rezago social, que tienen acceso muy limitado a energía poco fiable. La mayoría, como hemos señalado, vive en países no desarrollados (International Renewable Energy Agency, 2020a).

Tal escenario nos obliga a observar cómo se están tomando acciones para transformar esta matriz energética, sobre todo en los países no industrializados, y cómo, en última instancia, esta transformación coadyuva a combatir el rezago social (Naciones Unidas, 2019).

Y es que, paradójicamente, se estima que en 2030 en estos países se albergará la mitad de la infraestructura eólica instalada a escala mundial (Global Wind Energy Council, 2020). La Agencia Internacional de Energía Renovable (International Renewable Energy Agency, IRENA por sus siglas en inglés), afirma que en 2017 la capacidad eólica acumulada conectada a la red alcanzó 515 gigavatios (497 gigavatios de energía eólica terrestre y 18 gigavatios de energía eólica marina), lo que representó casi el 4 \% de la generación mundial de electricidad, y se pretende que para 2030 la cifra crezca a 839 gigavatios. Con ello, la generación de electricidad eólica en tierra aumentaría en casi un $65 \%$ a nivel mundial durante esta década (IRENA, 2020b).

En ese sentido, el gobierno mexicano ha apoyado la instalación de centrales eólicas en diferentes regiones del país bajo el auspicio de la Ley para el Aprovechamiento de Energías Limpias y el Financiamiento de la Transición Energética, la cual proyecta que en este país se deberá instalar tecnología que permita la generación de 25000 megavatios con fuentes eólicas para 2024 (Secretaría de Gobernación, 2015). Aunado a ello, y de acuerdo con la Agencia Mexicana de Energía Eólica (AMDEE), se espera que entre 2020 y 2022 la capacidad instalada permita la generación de los 25000 megavatios (JuárezHernández y León, 2014).

${ }^{1}$ Ver https://www.un.org/sustainabledevelopment/energy/ 
Sin embargo, más de 25 años después de la instalación de la primera central eólica en México se ha documentado una cantidad importante de conflictos socioambientales asociados a la puesta en marcha de esta clase de megaproyectos, lo que compromete su función de coadyuvar al combate al rezago social ya que se presenta lo que desde el Metabolismo Social (MS) se denomina "interacción biofísica desequilibrada" (Toledo, 2013).

En este marco, el objetivo central de nuestra investigación es analizar el combate al rezago social en la región del Istmo de Tehuantepec, Oaxaca, como resultado de la puesta en marcha de centrales eólicas y la propuesta de un nuevo índice de la percepción de mejora de la población respecto al combate al rezago social.

Para ello, dividiremos el análisis en cuatro apartados: en el primero presentamos las dimensiones analíticas holísticas propuestas por el MS para cuantificar las interacciones biofísicas entre sociedad y medio ambiente; en el segundo exponemos nuestra ruta metodológica y analizamos el contexto socioeconómico de cuatro municipios del Istmo donde se han instalado centrales eólicas, enfocándonos en el análisis del rezago social; en un tercer apartado presentamos los resultados de nuestro estudio arrojados por los SIG y la información recabada del Instituto Nacional de Estadística y Geografía (INEGI) y del Consejo Nacional de Evaluación de la Política de Desarrollo Social (Coneval); en un cuarto apartado aventuramos una propuesta metodológica que parte del MS, pero con un enfoque en los proyectos, lo que generó nuestra propuesta del índice de percepción local de mejora. Por último, aportamos algunas conclusiones sobre la investigación.

Pese a la necesidad de una transformación del modelo de generación energética a nivel mundial, el tránsito del antiguo esquema basado en la explotación/uso de energías fósiles ha dejado en herencia el viejo modelo económico altamente extractivo a los proyectos de generación energética vía renovables, que se consideran como una industria más y dejan de lado el beneficio ambiental, lo que ocasiona profundas afectaciones a las poblaciones locales y a las comunidades indígenas, que no son tomadas en cuenta en ninguna etapa de la toma de decisiones. Se produce, como veremos más adelante, una afectación profunda al paisaje y a la compleja y frágil biodiversidad de la región, lo que transforma los patrones de movilidad, de migración y de interrelación entre los ecosistemas que en la región conviven.

Por último, el alcance de esta investigación es la propuesta de un nuevo "índice local de percepción de mejora”, el cual se basa en el análisis del combate al rezago social desde la puesta en marcha de centrales eólicas en la región, el cual busca proporcionar una mejor referencia en la toma de decisiones en el futuro con proyectos similares, apoyando tanto a los promotores de dichos proyectos como a los habitantes afectados y a organizaciones encargadas de recibirlos, consumirlos y administrarlos.

\section{Análisis de la problemática socioambiental en el Istmo de Tehuantepec: una mirada desde el Metabolismo Social}

México cuenta con uno de los potenciales eólicos más altos a nivel mundial. De acuerdo con la IRENA, se estima que el potencial de nuestro país es superior a los 40000 megavatios. En la actualidad, hay una capacidad instalada de 185 megavatios, aunque se prevé que crezca rápidamente durante esta década. ${ }^{2}$

\footnotetext{
${ }^{2}$ Ver: https://maps.nrel.gov/rede-mexico/?aL=VOiAzp\%255Bv\%255D\%3Dt\%26sU8KUe\%255Bv\%255D\%3 Dt\%2 6sU8KUe\%
} 
Una de las regiones con mayor potencial es la del Istmo de Tehuantepec, en el estado de Oaxaca (ver Mapa 1), debido a que, por sus particularidades geográficas, la velocidad media anual de los vientos excede los 10 metros por segundo; en promedio, en el mundo se aprovechan vientos de 6.5 metros por segundo para la generación de energía (Juárez-Hernández y León, 2014). Esto se puede transformar en aproximadamente 1200 watts por metro cuadrado entre los meses de octubre y febrero (Secretaría de Gobernación, 2015).

\section{Mapa 1. Región del Istmo de Tehuantepec, Oaxaca}

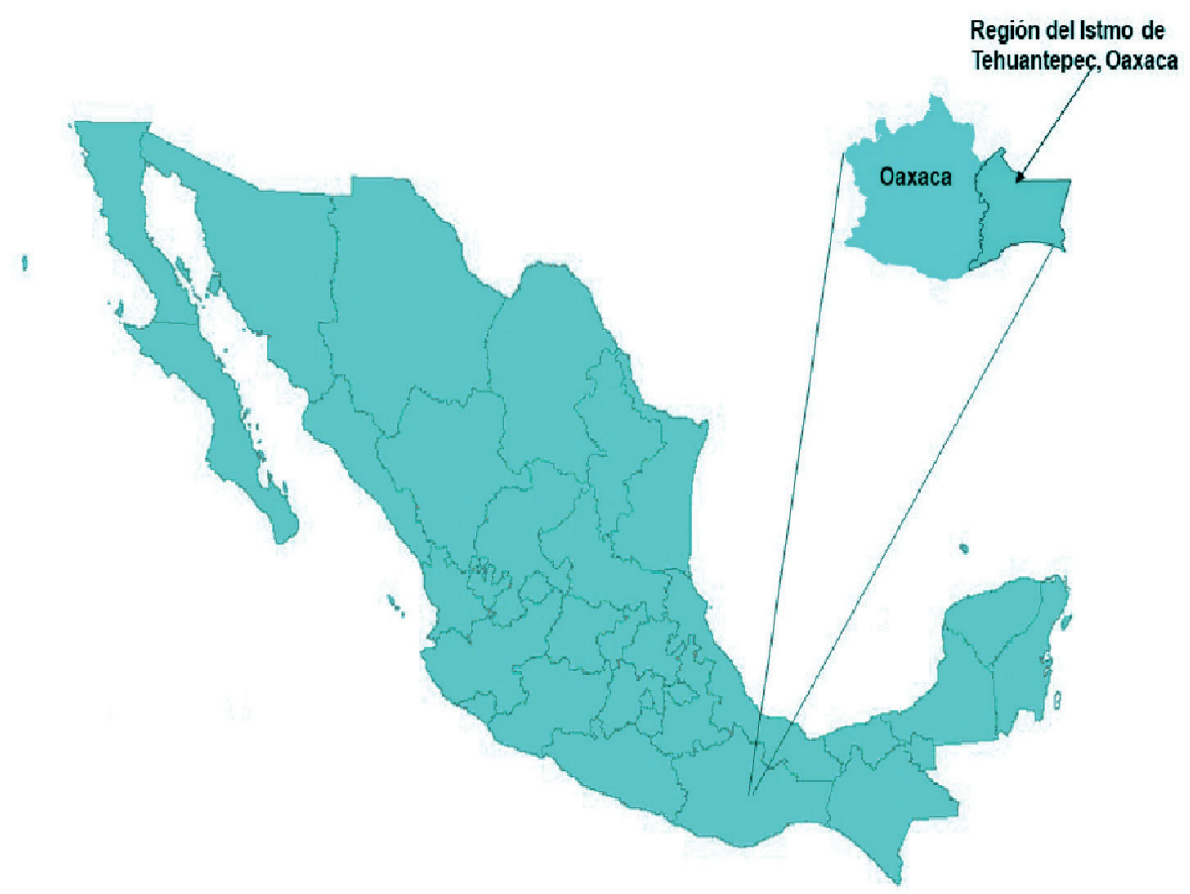

Fuente: elaboración propia con base en INEGI (2020) y Diario Oficial de la Federación (2020).

Esta región es la tercera franja de tierra más estrecha del continente americano después de Nicaragua y el Canal de Panamá, una franja de 200 kilómetros que conecta los océanos Pacífico y Atlántico. Esta estrechez hace que los vientos alcancen una gran fuerza y velocidad, lo que genera un "efecto túnel", del Atlántico hacia el Pacífico, por lo que se sostiene que hay capacidad de producción de energía eólica de 10000 megavatios en un área de 100000 hectáreas (Elliot et al., 2004).

El Istmo de Tehuantepec, por otro lado, tiene profundas raíces culturales representadas por los cinco grupos étnicos más importantes de la región (huaves, mixes, zapotecas, zoques y chontales), que suman cerca de 600000 habitantes, 15.7 \% de la población total del estado de Oaxaca (INEGI, 2010). Estos grupos poseen una cosmovisión particular sobre la tierra y el territorio, de tal modo que la tierra es observada como una unidad productiva y de consumo a la vez (Castillo, 2012).

255Bd\%255D $\% 3 \mathrm{D} 1 \& \mathrm{bL}=$ clight $\& \mathrm{cE}=0 \& \mathrm{lR}=0 \& \mathrm{mC}=24.410448 \% 2 \mathrm{C}-102.903647 \& \mathrm{zL}=5$ 
Este contexto puede ser analizado desde el MS, ya que esta corriente de pensamiento se ha desarrollado como una propuesta para conocer la relación que se gesta entre las culturas, la cosmovisión, los usos y las costumbres de las comunidades (Kaika y Swyngedouw, 2006), considerando asimismo el clima, la flora, la fauna, el suelo, el subsuelo y la biodiversidad, entre otros factores (Delgado, 2015).

El Metabolismo Social contiene dos esferas: una material, visible o tangible y otra inmaterial, invisible o intangible [...]. El Metabolismo Social comienza cuando los seres humanos socialmente agrupados se apropian materiales y energía de la naturaleza (input) y finaliza cuando depositan desechos, emanaciones o residuos en los espacios naturales (Toledo, 2013, p. 47).

Dentro de la esfera material o tangible, el MS postula que existen tres procesos de flujo de materiales o energía: los flujos de entrada (input), los flujos interiores y los flujos de salida (output). Además, el MS presenta cinco fenómenos tangibles: 1) la apropiación (A), 2) la transformación (T), 3) la distribución (D), 4) el consumo (C) y 5) la excreción (E) (Delgado, Campos y Rentería, 2012).

Para efectos de este estudio, en la Figura 1 mostramos una representación del proceso metabólico social para las energías renovables, específicamente las eólicas. En este diagrama buscamos reflejar el fenómeno de apropiación de la naturaleza respecto al viento y su uso energético. La transformación se refleja en la utilización de los aerogeneradores; la distribución se refiere a la red eléctrica por donde se traslada la energía; el consumo se refleja en los usuarios finales y en la industria; la excreción hace referencia a los desechos que se producen durante el proceso, es decir, la baja eficiencia energética, la pérdida de energía de forma innecesaria, transgresión al hábitat y emisiones de Gases de Efecto Invernadero (GEI). Esto último solo es posible si consideramos que la producción de aerogeneradores tiene una alta huella ecológica (Bohórquez y Güija, 2019) (ver Figura 1).

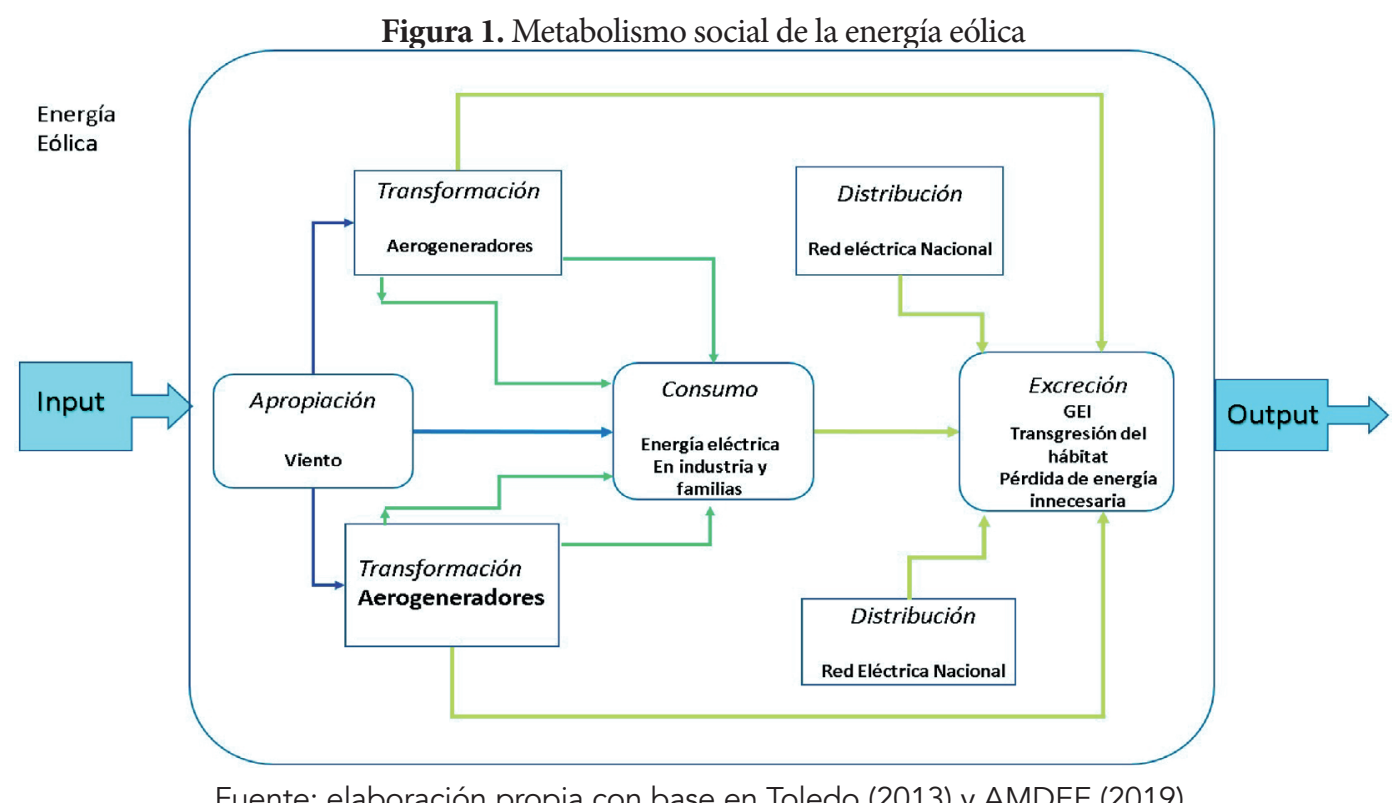

Fuente: elaboración propia con base en Toledo (2013) y AMDEE (2019). 
Por último, el MS que presentamos es una expresión simbólica alrededor del tema de las energías renovables. Buscamos explicitar cómo el concepto de MS puede ser utilizado en las diversas áreas de conocimiento, y en concreto consideramos representar los fenómenos y flujos que se derivan en un proyecto eólico (ver Figura 2): el flujo de entrada (input) es el proyecto eólico, la A es el territorio y recurso natural, y a su vez las flechas que van de la apropiación al consumo y a la excreción son: servicios e infraestructura y adquisición de bienes.

Figura 2. Metabolismo social en proyectos eólicos

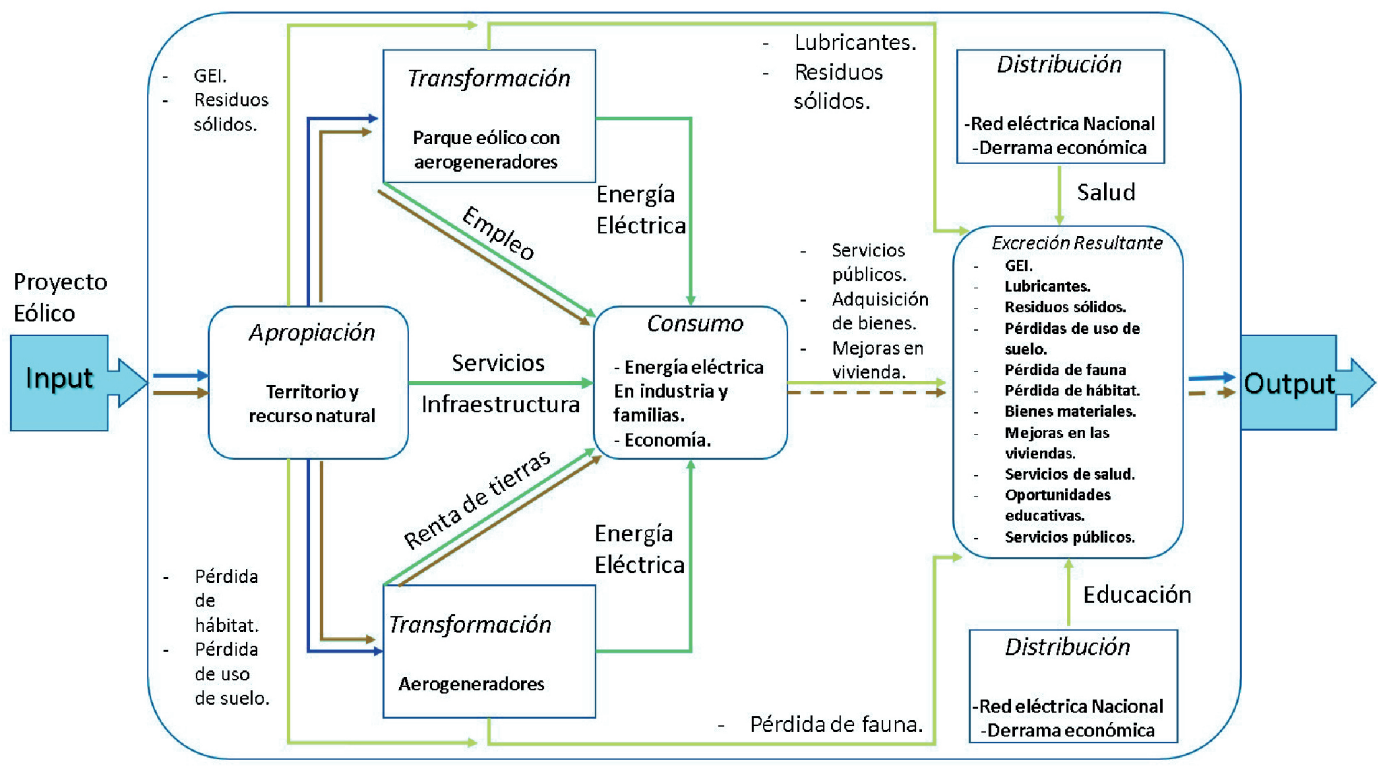

Fuente: elaboración propia con base en Toledo (2013) y AMDEE (2019).

En el caso de la Transformación (T), las flechas indican que el empleo, la tenencia de la tierra y la energía eléctrica son fundamentales para que se realice este proceso. Sin embargo, en este punto también hay posibilidades de generar excreciones (E), las cuales son consideradas como el producto resultante que puede ser funcional, reutilizable y residual positivo o negativo. Entre la Transformación y las Excreciones se encuentran las emisiones de gases de efecto invernadero y pérdida de uso de suelo, entre otros factores.

En cuanto a la distribución (D), los fenómenos que propicia son: acceso a la salud, acceso a la educación, energía eléctrica y economía; en caso de no existir distribución, habría una excreción por parte de la sociedad, es decir, que el Rezago Social (RS) aumentará. El flujo de salida (output) estaría conformado por los indicadores que analizamos para conocer el grado de RS en la unidad de análisis.

Por tanto, el enfoque integral que propone el MS permite comprender la naturaleza tras los proyectos eoloenergéticos en la región del Istmo, proyectos que, pese a partir de una noción de sostenibilidad, mantienen poca relación con el combate al RS que caracteriza a la región. 


\section{Metodología}

Para desarrollar la metodología del análisis del presente artículo se definió la unidad de estudio, el Istmo de Tehuantepec de Oaxaca, zona que está considerada como la de mayor potencial para generar energía eólica en México (Secretaría de Energía, 2018). Posteriormente, se ubicaron los municipios donde se localizan los parques eólicos: Asunción Ixtaltepec, El Espinal, Juchitán de Zaragoza, Santa María Xadani, Santo Domingo Ingenio y Unión Hidalgo (ver Cuadro 1).

Cuadro 1. Municipios de estudio en el Istmo de Tehuantepec, Oaxaca

\begin{tabular}{llcccccc} 
Nombre de los & $\begin{array}{c}\text { Núm. de } \\
\text { habitantes } \\
\mathbf{2 0 1 0}\end{array}$ & $\begin{array}{c}\text { Núm. de } \\
\text { habitantes } \\
\mathbf{2 0 1 5}\end{array}$ & $\begin{array}{c}\text { Índice de } \\
\text { rezago } \\
\text { social 2010 }\end{array}$ & $\begin{array}{c}\text { Índice de } \\
\text { rezago } \\
\text { social } \\
\mathbf{2 0 1 5}\end{array}$ & $\begin{array}{c}\text { Razón } \\
\text { de RS de } \\
\mathbf{2 0 1 0} \text { y } \\
\mathbf{2 0 1 5}\end{array}$ & $\begin{array}{c}\text { Capacidad } \\
\text { eólica } \\
\text { operativa } \\
\text { GWh/ a }\end{array}$ \\
\hline 1 & Asunción de Ixtaltepec & 14751 & 15105 & -0.6367 & -0.5967 & $-6.28 \%$ & 487.44 \\
\hline 2 & El Espinal & 8310 & 8575 & -1.2425 & -1.0965 & $-11.75 \%$ & 631.82 \\
\hline 3 & Juchitán de Zaragoza & 93038 & 98043 & -0.6310 & -0.6779 & $7.43 \%$ & 4395.93 \\
\hline 4 & Santa María Xadani & 7781 & 8795 & 0.6943 & 0.4122 & $-40.63 \%$ & 0 \\
\hline 5 & Santo Domingo & 7554 & 7965 & -0.4970 & -0.6152 & $23.78 \%$ & 1761.23 \\
\hline 6 & Unión Hidalgo & 13970 & 15347 & -0.8937 & -0.9031 & $1.05 \%$ & 304.34 \\
\hline
\end{tabular}

Fuente: elaboración propia con información del INEGI (2020) y Coneval (2019).

Los criterios para definir los municipios que se analizaron en el estudio fueron los siguientes: a) las entidades que contaran con mayor cantidad de habitantes, b) las localidades que presentaran el mayor crecimiento económico y c) los municipios que contaran con la mayor capacidad eólica instalada. Las entidades que cumplen con estos criterios son: Asunción Ixtaltepec, El Espinal, Juchitán de Zaragoza y Santo Domingo Ingenio, municipios que serán el centro del estudio (ver Cuadro 2); asimismo, elaboramos un mapa donde se representa cada municipio analizado dentro del Istmo de Tehuantepec (ver Cuadro 2).

Cuadro 2. Unidad de análisis

\begin{tabular}{|c|c|c|c|c|c|c|c|}
\hline & $\begin{array}{c}\text { Nombre de los } \\
\text { municipios }\end{array}$ & $\begin{array}{l}\text { Número } \\
\text { de } \\
\text { habitantes } \\
2010\end{array}$ & $\begin{array}{c}\text { Número } \\
\text { de } \\
\text { habitantes } \\
2015\end{array}$ & $\begin{array}{c}\text { Índice de } \\
\text { rezago } \\
\text { social } 2010\end{array}$ & $\begin{array}{c}\text { Índice de } \\
\text { rezago } \\
\text { social } 2015\end{array}$ & $\begin{array}{c}\text { Razón } \\
\text { de RS de } \\
2010 \text { y } \\
2015\end{array}$ & $\begin{array}{c}\text { Capacidad } \\
\text { eólica } \\
\text { operativa } \\
\text { GWh/ a }\end{array}$ \\
\hline 1 & $\begin{array}{l}\text { Asunción de } \\
\text { Ixtaltepec }\end{array}$ & 14751 & 15105 & -0.6367 & -0.5967 & $-6.28 \%$ & 487.44 \\
\hline 2 & El Espinal & 8310 & 8575 & -1.2425 & -1.0965 & $-11.75 \%$ & 631.82 \\
\hline 3 & $\begin{array}{l}\text { Juchitán de } \\
\text { Zaragoza }\end{array}$ & 93038 & 98043 & -0.6310 & -0.6779 & $7.43 \%$ & 4395.93 \\
\hline 4 & Santo Domingo & 7554 & 7965 & -0.4970 & -0.6152 & $23.78 \%$ & 1761.23 \\
\hline
\end{tabular}

Fuente: elaboración propia con información del INEGI (2020) y Coneval (2019). 
Por su parte, el Coneval diseñó una metodología que permite analizar el rezago social (RS), para lo cual considera once indicadores; 1) viviendas que no disponen de lavadora, 2) viviendas que no disponen de refrigerador, 3) población de 15 años o más analfabeta, 4) población de 6 a 14 años que no asiste a la escuela, 5) población de 15 años o más con educación básica incompleta, 6) población sin derechohabiencia o servicio de salud, 7) viviendas con piso de tierra, 8 ) viviendas que no disponen de agua entubada de la red pública, 9) viviendas que no disponen de drenaje, 10) viviendas que no disponen de excusado o sanitario y 11) viviendas que no disponen de energía eléctrica (Coneval, 2019).

Mapa 2. Unidades de análisis. Municipios de estudio en el Itsmo de Tehuantepec

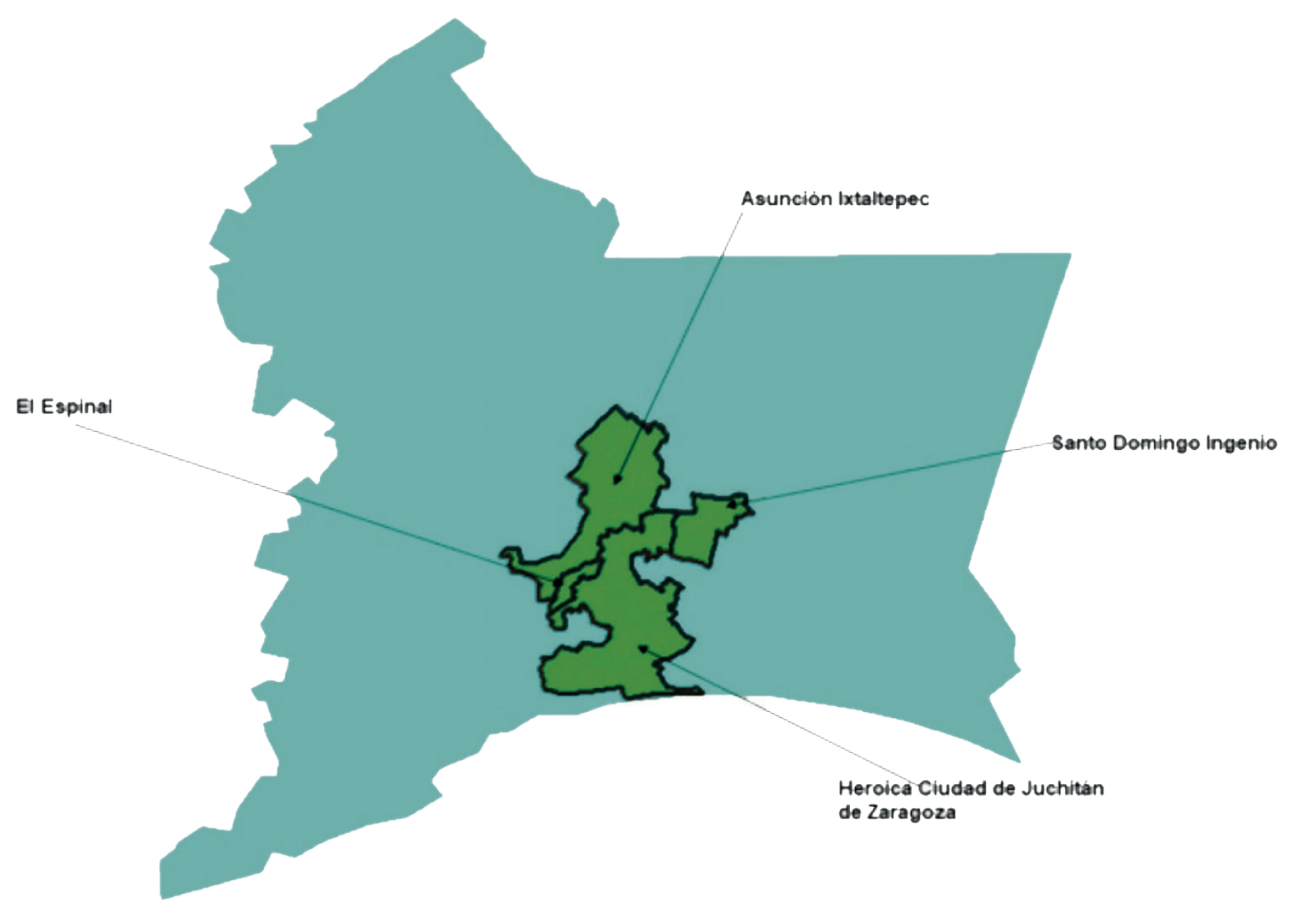

Fuente: elaboración propia con base en INEGI (2020).

Se realizó una diferenciación comparativa de los resultados obtenidos en los once indicadores para dos años de estudio, 2010 y 2015. Al conjunto de datos obtenidos por esta diferencia le llamaremos "variable de situación de mejora" en el caso de cada uno de los indicadores. Cuando el valor del indicador estudiado es positivo, implica una situación de mejora en el indicador en cuestión, mientras que cuando es negativo significa un retroceso en la característica medida por dicho indicador.

El promedio del conjunto de valores de la situación de mejora correspondiente para cada localidad brindará una referencia de cómo se percibe el cambio a lo largo del periodo de estudio. Sin embargo, con el fin de caracterizar aún más este número para la localidad durante el periodo de estudio, se propone dividir dicho promedio entre el máximo valor registrado en la variable de situación de mejora correspondiente a dicha localidad, para generar de esta manera un nuevo índice que denominamos 
"índice de percepción local de mejora”, el cual es una referencia numérica de cómo los individuos perciben el nivel de mejora promedio dentro de su localidad.

Los datos obtenidos representan el promedio general de mejora para el municipio en cuestión. Sin embargo, para obtener una referencia más local se divide el promedio entre el valor máximo de la diferencia de todos los indicadores. En el Cuadro 3 se presenta la medición del índice de percepción de mejora para el caso de Asunción Ixtaltepec, y de esa misma manera se replicó en los otros tres municipios (ver Cuadro 3).

Cuadro 3. Índice local de percepción de mejora

\begin{tabular}{|c|c|c|c|c|}
\hline \multicolumn{5}{|c|}{ Asunción de Ixtaltepec } \\
\hline & Indicadores & 2010 & 2015 & $\begin{array}{l}\text { Situación de mejora } \\
20102015\end{array}$ \\
\hline 1 & Viviendas que no disponen de lavadora & 43.99 & 32.09 & 11.90 \\
\hline 2 & Viviendas que no disponen de refrigerador & 22.11 & 15.78 & 6.33 \\
\hline 3 & Población de 15 años o más analfabeta & 16.08 & 13.96 & 2.12 \\
\hline 4 & $\begin{array}{l}\text { Población de } 6 \text { a } 14 \text { años que no asiste a la } \\
\text { escuela }\end{array}$ & 2.71 & 1.94 & 0.77 \\
\hline 5 & $\begin{array}{l}\text { Población de } 15 \text { años y más con educación } \\
\text { básica incompleta }\end{array}$ & 52.34 & 47.54 & 4.80 \\
\hline 6 & $\begin{array}{l}\text { Población sin derechohabiencia o servicios de } \\
\text { salud }\end{array}$ & 42.42 & 13.04 & 29.38 \\
\hline 7 & Viviendas con piso de tierra & 5.13 & 3.20 & 1.94 \\
\hline 8 & $\begin{array}{l}\text { Viviendas que no disponen de agua entubada } \\
\text { de la red pública }\end{array}$ & 5.58 & 2.83 & 2.75 \\
\hline 9 & Viviendas que no disponen de drenaje & 4.02 & 2.68 & 1.34 \\
\hline 10 & $\begin{array}{l}\text { Viviendas que no disponen de excusado o } \\
\text { sanitario }\end{array}$ & 2.98 & 1.77 & 1.21 \\
\hline 11 & Viviendas que no disponen de energía eléctrica & 2.22 & 1.64 & 0.58 \\
\hline \multicolumn{4}{|c|}{ Situación de mejora promedio } & 5.74 \\
\hline & & \multicolumn{2}{|c|}{$\begin{array}{l}\text { Índice local de percepción de } \\
\text { mejora }\end{array}$} & $19.53 \%$ \\
\hline
\end{tabular}

Fuente: elaboración propia con información del INEGI (2020) y Coneval (2019). 


\section{Análisis del rezago social de los municipios seleccionados}

El objetivo de este apartado es presentar gráficamente los resultados del análisis de los once indicadores de los municipios seleccionados para este estudio ubicados en la región del Istmo: Asunción Ixtaltepec, El Espinal, Juchitán de Zaragoza y Santo Domingo.

De acuerdo con datos de la Encuesta intercensal 2015 del INEGI, la población total del estado de Oaxaca es de 3967889 personas, de las cuales 52.4 \% son mujeres y 47.6 \% son hombres. Para el caso de estudio, el Istmo de Tehuantepec, el municipio de El Espinal cuenta con una población total de 8310 habitantes, Juchitán de Zaragoza con 93 038, Asunción Ixtaltepec con 14751 y Santo Domingo Ingenio con 7554 habitantes. En la región hay casi 150000 habitantes de origen indígena (INEGI, 2015).

A continuación, presentamos una comparación de los datos estadísticos para estos cuatro municipios para los años 2010 y 2015 con el fin de generar una perspectiva sobre la evolución del RS de dichas comunidades y proyectar una perspectiva global que permita entender el impacto que han tenido los proyectos eoloenergéticos en estos municipios. Para ello, seguimos la definición propuesta por Coneval para caracterizar el rezago social, apoyándonos en los datos arrojados por dicha institución para el periodo 2010-2015.

Para el Coneval (2019), el índice de rezago social parte del concepto multidimensional que establece sobre pobreza; en este sentido, para este organismo la pobreza es aquella situación en la que un individuo presenta al menos uno de los nueve indicadores de carencia social - ingreso corriente per cápita, rezago educativo promedio en el hogar, acceso a los servicios de salud, acceso a la seguridad social, calidad y espacios de la vivienda, acceso a una alimentación nutritiva y de calidad, servicios básicos en la vivienda, grado de cohesión social y grado de accesibilidad a carretera pavimentada-. La pobreza es analizada entonces por este organismo como una problemática multidimensional, es decir, como resultado de varias dimensiones o factores económicos y sociales en su concepción y definición.

De acuerdo con esta construcción de la noción de pobreza multidimensional, el Coneval construye el concepto de índice de rezago social incorporando cuatro indicadores: educación, acceso a servicios de salud, servicios básicos y espacios de vivienda y activos en el hogar. Esta noción es observada en los tres niveles de agregación geográfica (estatal, municipal y local) y es elaborada bajo la técnica estadística de componentes principales, hecho que posibilita resumir diferentes dimensiones del fenómeno de estudio en un indicador agregado.

A continuación presentamos las gráficas que explican el análisis del índice de RS en los municipios de Asunción Ixtaltepec, El Espinal, Juchitán de Zaragoza y Santo Domingo Ingenio. En ellas realizamos el comparativo de los años 2010 y 2015 tomando como punto de partida el indicador once que está enfocado en conocer el número de viviendas que no disponen de energía eléctrica. Esto en razón de que el estudio versa sobre el análisis del combate del RS en el Istmo de Tehuantepec en los años 2010 y 2015 como resultado de la puesta en marcha de parques eólicos.

En el caso del municipio de Asunción Ixtaltepec (ver Gráfica 1), los indicadores uno, dos y cinco que se refieren a la ausencia de lavadora, ausencia de refrigerador, así como a la población de 15 años y más con educación básica incompleta, mostraron una mejora entre 2010 y 2015. Sin embargo, el indicador once, concerniente a la existencia de energía eléctrica en las viviendas, tuvo una mejora mínima en el mismo periodo, lo cual indica que este indicador es poco relevante para impulsar el crecimiento de los 
indicadores uno y dos. Consideramos que existió un factor más allá de la disponibilidad del recurso energético eléctrico, el cual impactó de forma decisiva en la adquisición de estos electrodomésticos. Impactó también en las personas mayores de 15 años para que lograran terminar su educación básica durante el periodo de estudio, lo cual lleva implícito un factor de carácter económico, y se traduce en que entre 2010 y 2015 las familias tuvieron una mejora económica que les permitió adquirir nuevos bienes y alcanzar un mejor nivel educativo. Por tanto, para este municipio, la percepción local de bienestar promedio mejoró en un 19.53 \% durante el periodo de estudio. Sin embargo, esto coloca a Asunción Ixtaltepec en el último lugar entre los municipios analizados basándonos en el criterio formado por el índice local de percepción de mejora.

Gráfica 1. Asunción Ixtaltepec. Comparativo de rezago social 2010/2015

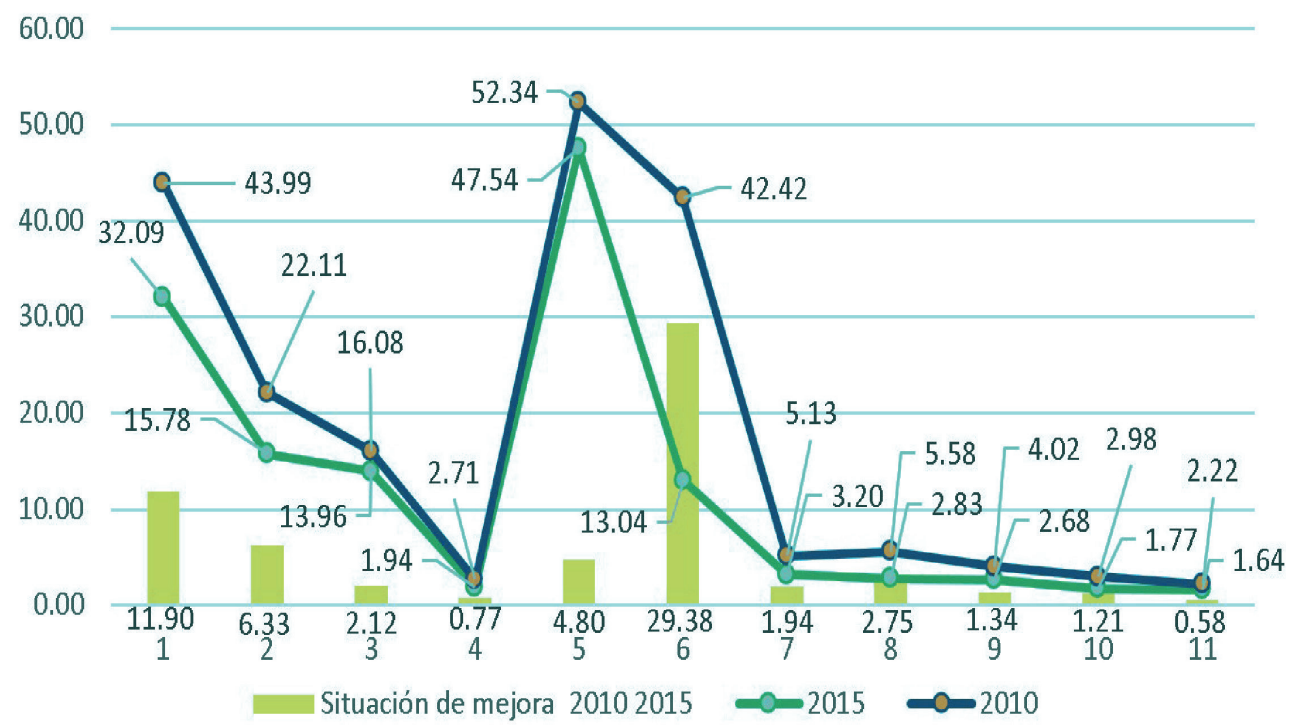

Fuente: Elaboración propia con información del INEGI (2020) y Coneval (2019).

Respecto al municipio de El Espinal (ver Gráfica 2), los indicadores ocho y seis que se refieren a disponibilidad de agua entubada en la vivienda y a la población sin derechohabiencia a servicios de salud fueron los que presentaron una mejora significativa, seguidos por el indicador número cinco - población de 15 años y más con educación básica incompleta-durante el periodo de 2010 a 2015. Seguían a estos los indicadores dos y uno, que contemplan los bienes electrodomésticos presentes en la vivienda como refrigerador y lavadora. En lo que corresponde al indicador once — viviendas que no disponen de energía eléctrica一, se ve reflejada una mejora del $1.28 \%$, que en apariencia tiene mayor relación con los indicares uno y dos, que presentan una mejora del $2.45 \%$ y $2.87 \%$ respectivamente. Ello muestra que el incremento pudo estar impulsado por una posible mejora económica en los ingresos de la población, lo que a su vez implica una mejora en el nivel educativo de la población que se refleja, este último, a través del indicador cinco. 
Gráfica 2. El Espinal. Comparativo de rezago social 2010/2015

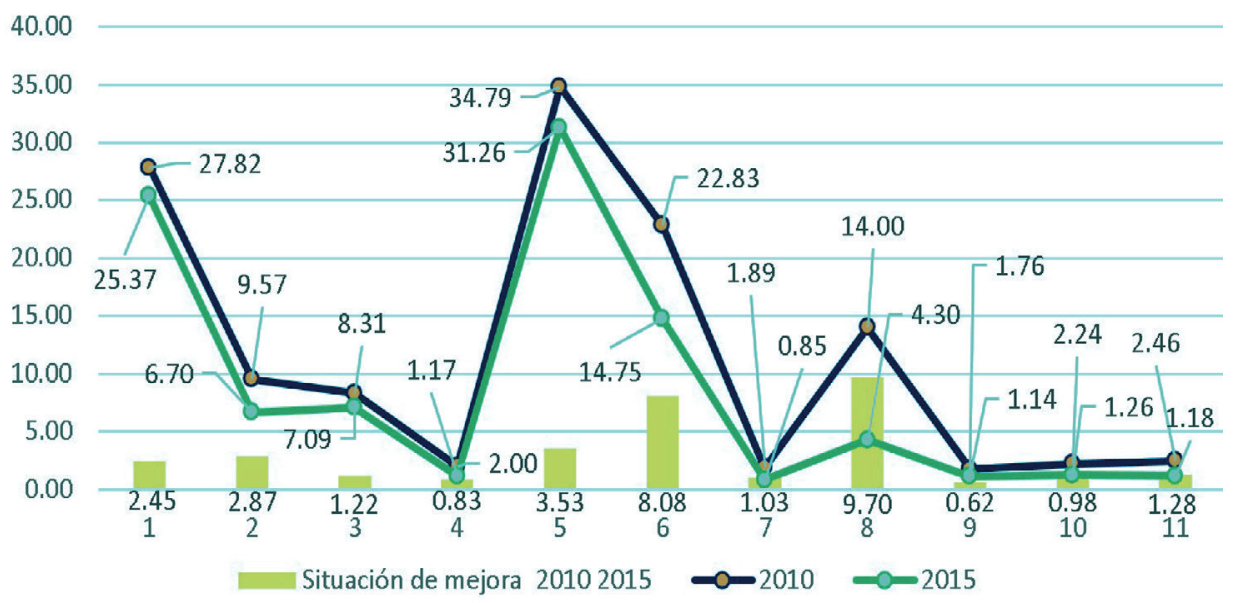

Fuente: Elaboración propia con información del INEGI (2020) y Coneval (2019).

En el caso de este municipio, la percepción local de mejora en su bienestar promedio alcanza el $30.55 \%$ en 2015 respecto de su situación en 2010. Cabe mencionar que El Espinal, bajo este criterio, se coloca como el municipio mejor evaluado en cuanto a la percepción local de mejora.

En cuanto al municipio de Juchitán de Zaragoza (ver Gráfica 3), los indicadores seis y dos, que se refieren a la población sin derechohabiencia a servicios de salud (26.85\%) y viviendas que no disponen de refrigerador $(7.05 \%)$ respectivamente, fueron los que presentaron una mejora, seguidos por los indicadores cinco — población de 15 años y más con educación básica incompleta (7.00 \%) — y uno —viviendas que no disponen de lavadora (4.98\%)-.

Gráfica 3. Juchitán de Zaragoza. Comparativo de rezago social 2010/2015

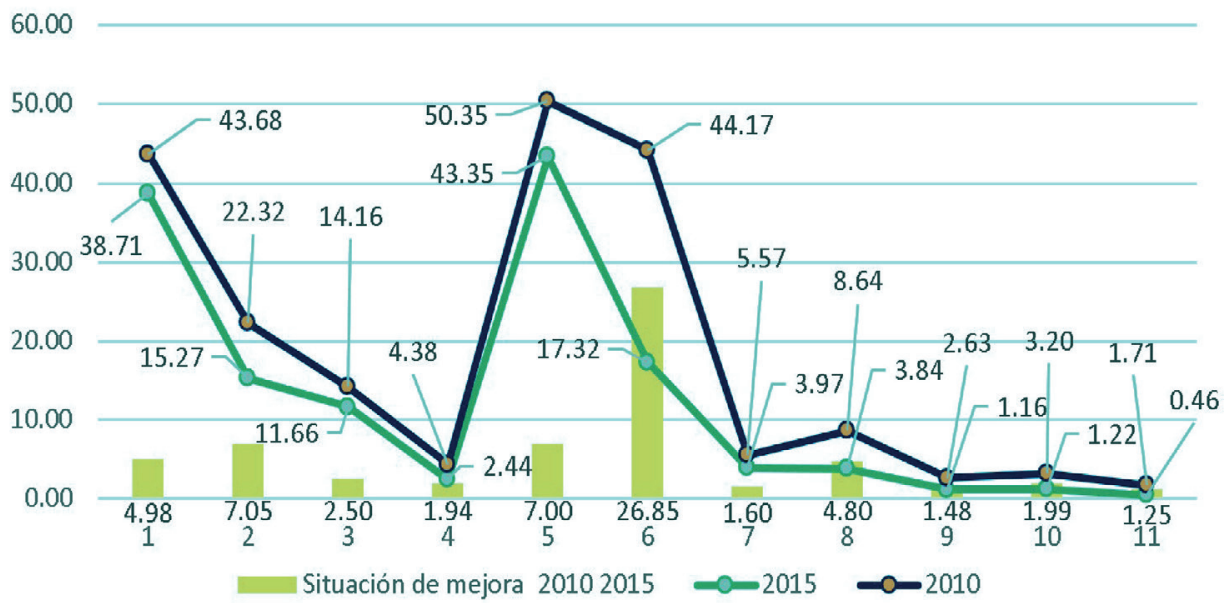

Fuente: elaboración propia con información del INEGI (2020) y Coneval (2019). 
Finalmente, el indicador ocho, correspondiente a la disponibilidad de agua entubada en las viviendas, presenta mayor relevancia. En lo que corresponde al indicador once - viviendas que no disponen de energía eléctrica一, se ve reflejada una mejora del $1.25 \%$, que en apariencia para este municipio no tiene una relación proporcional con los indicares uno y dos, los cuales presentan una mejora del 4.98 \% y el $7.05 \%$; esto muestra que el crecimiento de dichos indicadores podría estar soportado por una mejora económica en los ingresos de las viviendas, lo que pudo implicar una mejora en el nivel educativo de la población y reflejarse a través del indicador cinco.

Para el caso de este municipio, la percepción local de mejora en su bienestar promedio alcanzó el $20.80 \%$ en 2015 respecto de su situación en 2010, por lo que esta localidad ocupa el tercer lugar de este estudio.

En el caso de Santo Domingo Ingenio (ver Gráfica 4), nuevamente los indicadores ocho y seis, relacionados con agua potable y derechohabiencia, tienen una tendencia de mejora relevante sobre los otros en un $25.96 \%$ y $24.15 \%$, seguidos por los indicadores uno y dos, que reflejan un aumento en la existencia de electrodomésticos en las viviendas, $10.47 \%$ para lavadoras y $6.70 \%$ para refrigeradores.

Gráfica 4. Santo Domingo Ingenio. Comparativo de rezago social 2010/2015

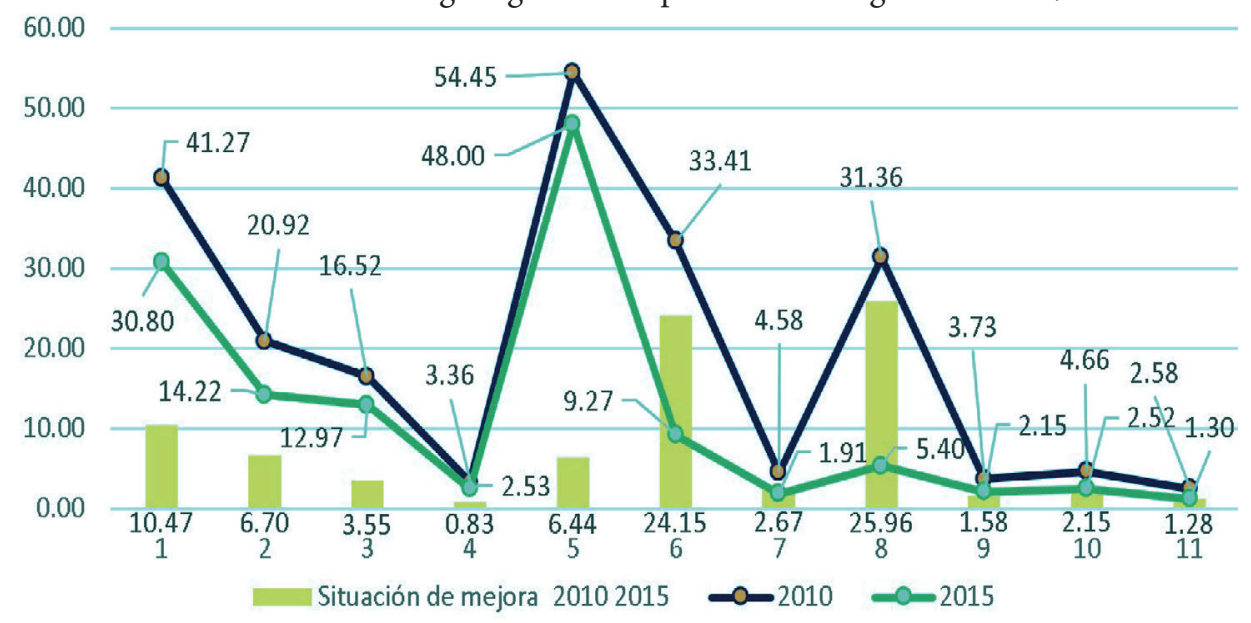

Fuente: elaboración propia con información del INEGI (2020) y Coneval (2019).

Como punto relevante queda el indicador número cinco, que muestra la disminución de la población con educación básica incompleta, el cual refleja una mejora del 6.44\%. Finalmente, el indicador de interés, el número once, muestra una reducción del $1.28 \%$ en las viviendas que no disponen de energía eléctrica, lo cual confirma que no existe una relación proporcional entre la adquisición de electrodomésticos y la disponibilidad de la energía eléctrica en las viviendas, y refleja que hay un motor económico que impulsa la adquisición de bienes y que posiblemente esté ligado también a una mejora en la situación del nivel educativo presente en las localidades de estudio.

Por otro lado, para los pobladores de Santo Domingo Ingenio la percepción local promedio de mejora en sus condiciones de bienestar observadas puede referenciarse mediante el índice local de percepción de mejora propuesto, con un valor del $30.04 \%$, siendo este municipio el segundo con mejor evaluación local en cuanto a su mejora en rezago social en 2015 respecto a 2010. 
Finalmente, podemos sintetizar que la percepción de mejora de la unidad de análisis, como se presenta en el Mapa 3, expone en cifras cerradas que en el caso de Santo Domingo la percepción es del 20 \%, El Espinal experimentó una mejora del 31 \%, Juchitán demarcó un 21 \% y Santo Domingo Ingenio el $30 \%$, referidos al año 2015.

Mapa 3. Percepción de mejora en unidades de análisis, municipios de estudio

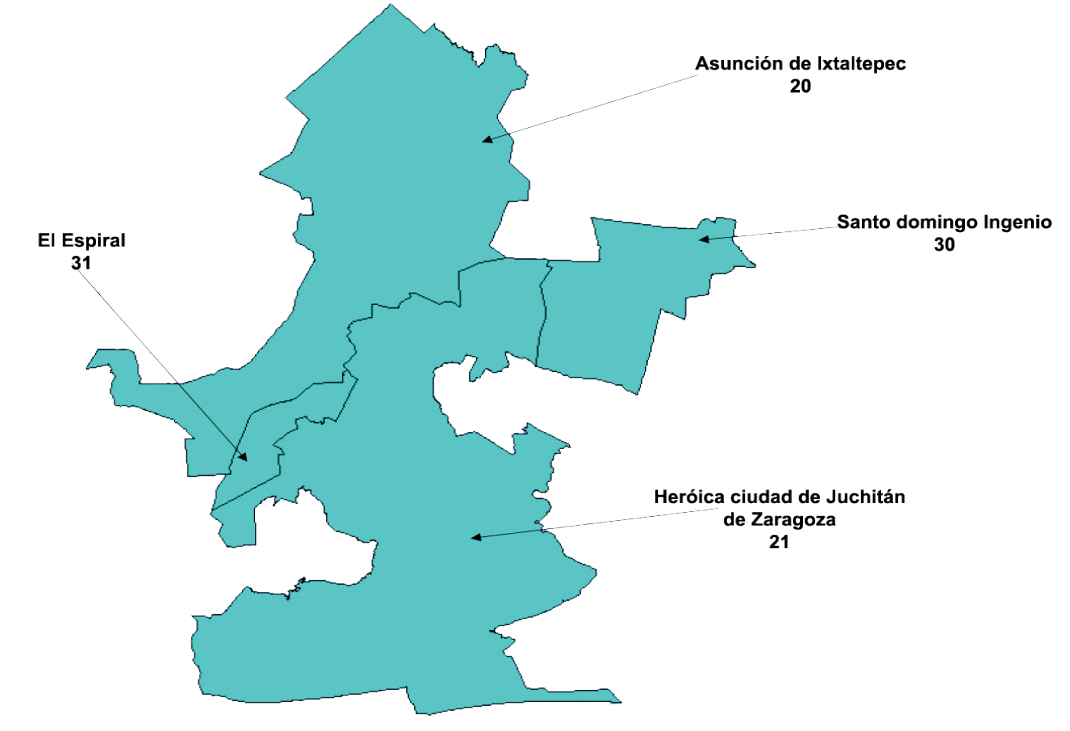

Fuente: elaboración propia con información del INEGI (2020) y Coneval (2019).

\section{Discusión}

Con la información actual tenemos la oportunidad de identificar las cinco etapas definidas por la teoría de MS.

A través del análisis fue posible identificar de manera indirecta una mejora en la economía de las localidades estudiadas que muy probablemente deriva de la derrama económica que generan estos proyectos tal cual se espera como resultante de la Apropiación, según la perspectiva teórica del MS, así como por parte de la Transformación.

Sin embargo, tal cual lo prevé la teoría, existen excreciones del sistema que no necesariamente son desechos directos al medio ambiente, sino productos resultantes del consumo y distribución que se quedan y conviven con la comunidad en Consumo y que provienen de la Apropiación, la Transformación y la Distribución; estas excreciones o productos, para nuestros casos de estudio, son los once indicadores de rezago social y se explican a continuación.

Cuando inicia el proyecto, conlleva un accionamiento a diferentes niveles administrativos y una derrama económica tanto privada como municipal, de tal forma que se genera una Apropiación cuyos productos son principalmente infraestructura y extensión de servicios públicos para recibir los proyectos, y que además son consumidos por las localidades en Consumo. 
Por su parte, la Transformación genera productos para consumo así como empleos directos e indirectos, y promueve la venta o arrendamiento de tierras en sus etapas iniciales, que también son consumidas por las localidades en Consumo, lo que aumenta los ingresos de las viviendas en las localidades de estudio. Esto, por su parte, puede ser medido de forma indirecta a través de los bienes materiales que consumen, que para Coneval (2019) e INEGI (2020) son lavadoras y refrigeradores.

Finalmente, se esperaría que el producto de la Transformación fuese consumido en la etapa de Consumo y que promoviera el crecimiento positivo de al menos un indicador. Como parte de todo el ciclo de consumo implicado en Transformación y la distribución tanto de los bienes de consumo como de la derrama económica que conlleva, la etapa de Distribución brinda como excreción resultante mejoras en los servicios de salud y en las oportunidades de educación. De esta forma, las excreciones resultantes para el diagrama propuesto corresponden de manera puntual con los indicadores disponibles en la información analizada.

Una vez realizado el análisis de la sección 3 con base en la información obtenida del Coneval y de INEGI, y habiendo cotejado esta información con la teoría de MS, como se ha descrito anteriormente, apoyándonos en la información de la Figura 1, es evidente que, debido a su naturaleza e intención de origen, la metodología implementada por Coneval contempla únicamente indicadores de carácter social; ello acota la visión completa de los impactos percibidos por las localidades, cuando diferentes proyectos energéticos llegan a esas regiones, principalmente en el aspecto ambiental.

Debido a esto, deseamos proponer un diagrama de MS (ver Figura 3) donde se complementa el contenido de la Figura 1 con las principales excreciones de carácter ambiental que deberían tomarse en cuenta por las instituciones mencionadas para dimensionar de una mejor manera los impactos de los proyectos relacionados con energías renovables, tanto en lo concerniente a RS, como en la calidad de vida de los habitantes y la salud ambiental de la región.

Figura 3. Metabolismo social para proyectos de energías renovables

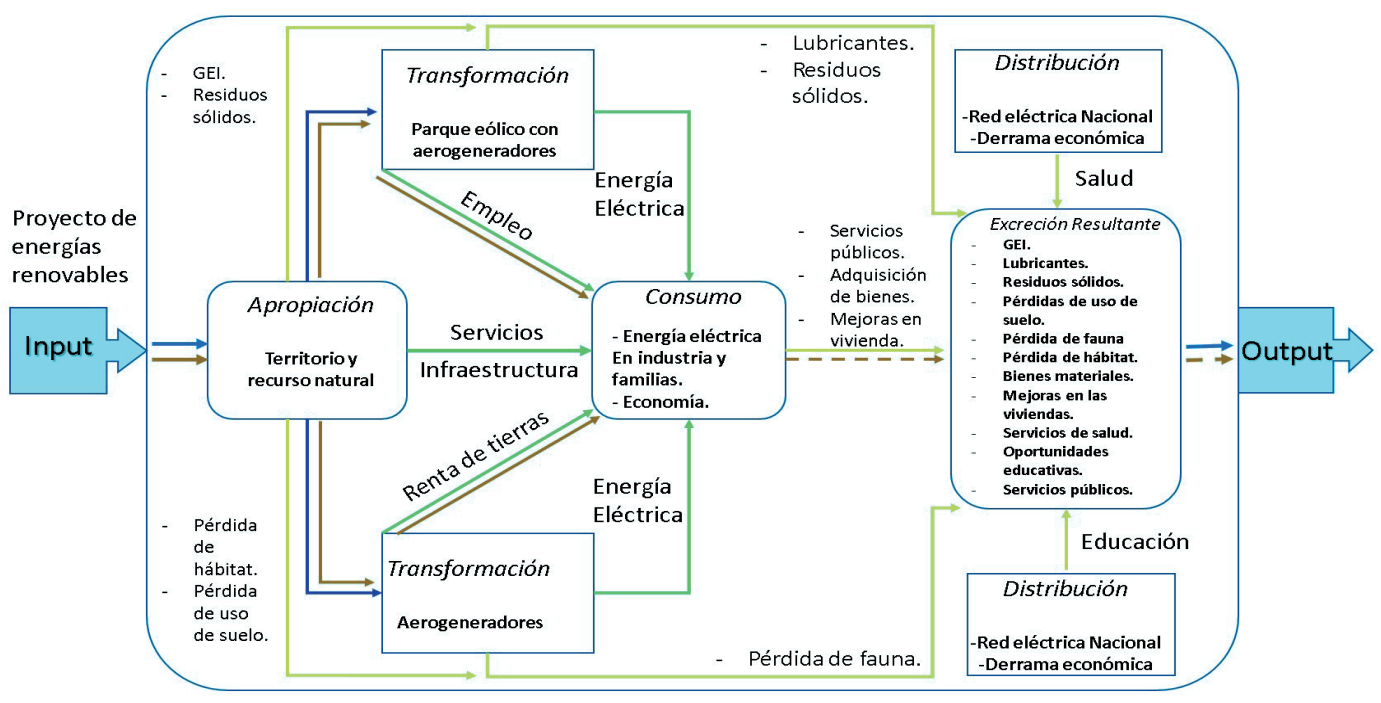

Fuente: elaboración propia con base en Toledo (2013). 


\section{Conclusiones finales}

Los rezagos socioeconómicos en los municipios analizados en esta investigación persisten, aunque han mostrado una mejora que al parecer se origina desde la parte económica; sin embargo, es un hecho que la región del Istmo de forma general se encuentra en los niveles de vulnerabilidad social más altos a nivel nacional.

Buscamos con este trabajo analizar cómo algunos proyectos eólicos han servido para coadyuvar al combate del rezago social en la región del Istmo, mientras que otros no lo han hecho, sin que ello nos permita hacer una injusta generalización. Escapa a los alcances de esta investigación analizar la diversidad de proyectos eólicos que existen en la región, así como de los proyectos de energías alternativas en general, diversidad que responde a la naturaleza misma de estos proyectos (públicos, privados o alianzas público/privadas), así como a las disputas tecnológicas que existe tras los proyectos de esta naturaleza y magnitud (Grunstein, 2016), hecho que incluso ha jugado en contra de la transición energética y el cambio de modelo de generación al que estos proyectos deberían apuntar (Castillo, 2012; Zárate y Fraga, 2016).

Son evidentes por el momento algunas circunstancias como las siguientes: no consideran en ninguna etapa la toma de decisiones de las poblaciones afectadas, no generan empleos más allá de los temporales en su construcción, y afectan decisivamente al paisaje, a las tierras, al agua y a flora y fauna de la región, sin que existan hasta el momento estudios especializados que muestren en profundidad la manera en la que los aerogeneradores están transformando los patrones de movilidad, de migración y de interrelación entre los ecosistemas que en la región conviven.

Es por eso que proponemos la creación y utilización de un nuevo índice que llamamos "índice local de percepción de mejora" (ver Cuadro 3) basado en la información actual disponible en INEGI y Coneval, pero tratada según la metodología que proponemos. Dicho índice proporcionará una mejor referencia en la toma de decisiones a futuro con proyectos similares, apoyando tanto a los promotores de dichos proyectos como a los habitantes y organizaciones encargados de recibirlos, consumirlos y administrarlos.

El conflicto socioambiental en el Istmo de Tehuantepec revela que, si bien la energía eólica debería plantearse en las políticas públicas a futuro en materia de generación de energía en afán de reducir la huella ecológica, tanto su planeación como su gestión se definen por la obtención de ganancias a corto plazo y no por un interés real por mitigar los efectos negativos del cambio climático relacionados con la generación de los GEI (Bohórquez y Güiza, 2019; Castillo, 2012; Juárez-Hernández y León, 2014) Por ello, los criterios para determinar la viabilidad de los parques eólicos en términos socioambientales no deben restringirse a la potencial reducción de emisiones de GEI, mucho menos a los criterios de los Mecanismos de Desarrollo Limpio (MDL).

Como una segunda propuesta, y basados en la teoría de Metabolismo Social (MS), generamos un diagrama general de impacto socioambiental que debería ser tomado en cuenta, y además proponemos el tipo de indicadores que debería medirse para tener una referencia completa del impacto implicado en las regiones donde se implementen proyectos de carácter energético enfocados en energías renovables (ver Figura 3).

Por último, el análisis de los municipios que conforman la región demostró que la implementación de estos megaproyectos constituye un factor medible indirectamente, favorable para el desarrollo de la región, aunque los beneficios económicos no han impactado como podría esperarse en el nivel de vida de las personas a las que les afectó la construcción de los aerogeneradores en sus terrenos y la accesi- 
bilidad de la energía eléctrica para los habitantes ha mejorado mínimamente. Nos parece fundamental que la implementación de proyectos de esta naturaleza tiene que estar forzosamente respaldado por la legitimidad social para asegurar la distribución equitativa de sus beneficios económicos y ambientales, por lo que debe cambiar el modelo bajo el cual han sido implementados (extractivo), el cual reproduce y profundiza el rezago social. Para ello, las decisiones deben estar basadas en la información y en el diálogo de saberes, intercambio que involucra, en un ejercicio de gobernanza, al Estado, al sector productivo, a las universidades, a grupos defensores y, sobre todo, a las comunidades.

Por ello, de acuerdo con la tendencia de la nueva narrativa ambiental en su apuesta por una transformación del modelo de generación energética a nivel mundial por las energías renovables, remarcamos la necesidad de abandonar el antiguo esquema basado en la extracción intensiva e invasiva de recursos heredada del modelo económico dominante, el cual observa a las renovables como una industria más y deja de lado el beneficio ambiental, lo que ocasiona profundas afectaciones a las poblaciones locales y a las comunidades indígenas.

Estamos seguros de que nuestra metodología será un nuevo auxiliar en análisis posteriores que competen al área socioambiental al considerar otros elementos como las afectaciones profundas al delicado equilibrio biosistémico de la región, a los problemas asociados a la salud humana y a las repercusiones socioeconómicas, de forma tal que, en el tránsito hacia un modelo energético alternativo, las repercusiones socioambientales se minimicen y los beneficios ecológicos contemplen también un diálogo de saberes con las comunidades.

\section{Referencias}

Asociación Mexicana de Energía Eólica. (2019). Buenas prácticas 2018. https://amdee.org/Publicaciones/ BuenasPracticas_final_16_10_18.pdf

Bohórquez, Y., y Güiza, S. (2019). Análisis de los impactos ambientales y sociales de los aerogeneradores a través del análisis del ciclo de vida apoyado en el software Open LCA. Universidad de La Salle.

Castillo, E. (2012). Problemática en torno a la construcción de parques eólicos en el Istmo de Tehuantepec. Desarrollo Local Sostenible, 4(12), 1-14.

Consejo Nacional de Evaluación de la Política de Desarrollo Social. (2019). Metodología para la medición multidimensional de la pobreza en México. https://www.coneval.org.mx/Medicion/MP/Paginas/ Metodologia.aspx

Delgado, G. C. (2015). Complejidad e interdisciplina en las nuevas perspectivas socioecológicas: la ecología política del metabolismo urbano. Letras Verdes. Revista Latinoamericana de Estudios Socioambientales, 17, 108-130. https://doi.org/10.17141/letrasverdes.17.2015.1442

Delgado, G. C., Campos, C., y Rentería P. (2012). Cambio climático y el metabolismo urbano de las Megaurbes Latinoamericanas. Hábitat Sustentable, 2(1), 2-25. http://revistas.ubiobio.cl/index.php/RHS/article/ view/409

Diario Oficial de la Federación. (2020). Programa regional. Corredor Interoceánico del Istmo de Tehuantepec. Secretaría de Gobernación. https://www.dof.gob.mx/nota_detalle.php? codigo=5597712\&fecha=04/08/2020

Elliot, D., Schwartz, M., Scott, G., Haymes, S., Heimiller, D., y George, R. (2004). Atlas de recursos eólicos del estado de Oaxaca. Laboratorio Nacional de Energía Renovable. http://www.nacionmulticultural.unam.mx/ mezinal/docs/330.pdf

Global Wind Energy Council. (2020). Global Wind Report 2019. https://gwec.net/global-wind-report-2019/

Grunstein, M. (2016). Contra el viento: regulación, crisis social y cambio institucional en el Corredor Eólico del Istmo. Economía, Sociedad y Territorio, 16(51), 485-517. http://www.scielo.org.mx/pdf/est/v16n51/24486183-est-16-51-00485.pdf

House of Commons. (2010). Energy and Climate Change Committee. Fifth Report of Session 2009-2010. Fuel poverty. https://publications.parliament.uk/pa/cm200910/cmselect/cmenergy/424/42402.htm 
Instituto Nacional de Estadística y Geografía. (2010). Censo de Población y Vivienda 2010. https://www.inegi. org.mx/programas/ccpv/2010/default.html\#Datos_abiertos

Instituto Nacional de Estadística y Geografía. (2015). Encuesta intercensal 2015. https://www.inegi.org.mx/ programas/intercensal/2015/

Instituto Nacional de Estadística y Geografía. (2020). Mapa digital de escritorio.

International Renewable Energy Agency. (2020a). Renewable Energy and Climate Pledges: Five Years after the Paris Agreement. https://www.irena.org/publications/2020/Dec/Renewable-energy-and-climate-pledges

International Renewable Energy Agency. (2020b). Renewable Capacity Statistics 2020. https://www.irena.org/ publications/2020/Mar/Renewable-Capacity-Statistics-2020

Juárez-Hernández, S., y León, G. (2014). Energía eólica en el Istmo de Tehuantepec: desarrollo, actores y oposición social. Problemas del Desarrollo, 178(45), 139-162.

Kaika, M., y Swyngedouw, E. (2006). Urban political ecology: politicizing the production of urban natures. En T. Perreault (ed.), Handbook of Political Ecology (pp. 609-619). Routledge.

Kozulj, R. (2009). Contribución de los servicios energéticos a los Objetivos del Desarrollo del Milenio y a la mitigación de la pobreza en América Latina y el Caribe. Comisión Económica para América Latina y el Caribe; Sociedad Alemana de Competencia Técnica; Programa de Naciones Unidas para el Desarrollo.

Naciones Unidas. (2019). 2018 Energy Statistics Yearbook. Naciones Unidas-Departamento de Asuntos Económicos y Sociales. https://unstats.un.org/unsd/energystats/pubs/yearbook/

Secretaría de Gobernación. (2015). La energía eólica en México. Secretaría de Gobernación; Comisión para el Diálogo con los Pueblos Indígenas de México.

Secretaría de Energía. (2018). Balance nacional de energía. https:/www.gob.mx/sener/documentos/balancenacional-de-energia-2018

Toledo, V. (2013). El metabolismo social: una nueva teoría socioecológica. Relaciones, 136, 41-71.

Zárate, E., y Fraga, J. (2016). La política eólica mexicana: controversias sociales y ambientales debido a su implementación territorial. Estudios de caso en Oaxaca y Yucatán. Trace, 69, 65-95. https://journals. openedition.org/trace/2137 